\title{
Relics of Primordial Star Formation: The Milky Way and Local Dwarfs
}

\author{
Timothy C. Beers ${ }^{1}$, Young Sun Lee ${ }^{1}$, and Daniela Carollo ${ }^{2}$ \\ ${ }^{1}$ Department of Physics \& Astronomy, CSCE: Center for the Study of Cosmic Evolution, and \\ JINA: Joint Institute for Nuclear Astrophysics, Michigan State University, \\ East Lansing, MI 48824, USA \\ email: beers@pa.msu.edu, lee@pa.msu.edu \\ ${ }^{2}$ Research School of Astronomy and Astrophysics, Australian National University, Mount \\ Stromlo Observatory, Cotter Road, Weston, ACT 2611, Australia, and INAF-Osservatorio \\ Astronomico di Torino, 10025 Pino Torinese, Italy, \\ email: carollo@mso.anu.edu.au
}

\begin{abstract}
Massive spectroscopic surveys of stars in the thick disk and halo populations of the Galaxy hold the potential to provide strong constraints on the processes involved in (and the timing of) the assembly history of the primary structural components of the Galaxy. In this talk, we explore what has been learned from one of the first such dedicated surveys, SDSS/SEGUE. Over the course of the past three years, SEGUE has obtained spectra for over 200,000 stars, while another hundred thousand stars been added from the calibration star observations of the (primarily extragalactic) SDSS, and other directed programs. A total of well over 10,000 stars with $[\mathrm{Fe} / \mathrm{H}]<-2.0$ have been discovered, including several hundred with $[\mathrm{Fe} / \mathrm{H}]<-3.0$. Their kinematics have revealed a inner/outer halo structure of the Galaxy.

New determinations of the alpha element ratios for tens of thousands of these stars are reported. Correlations of the alpha-element ratios with kinematics and orbital parameters can be used to test models of the likely formation of the thick-disk and halo components. These new data will (eventually) be considered in connection with possible associations with the present dwarf satellite galaxies of the Milky Way.
\end{abstract}

Keywords. Astronomical data bases: surveys, techniques: spectroscopic, methods: data analysis, stars: fundamental parameters, Galaxy: disk, Galaxy: halo

\section{Introduction}

The modern picture of galaxy formation, as described in the context of the $\Lambda$ CDM paradigm, suggests that large galaxies like the Milky Way are formed from a series of mergers involving the mergers of dark matter dominated sub-haloes with a mass distribution set by the initial conditions that emerged from the Big Bang. These initial mergers are followed by prolonged (continuing to the present) accretion of numerous lower mass sub-haloes, likely in a non-dissipative manner (e.g., Bekki \& Chiba 2001). The process is expected to leave behind many hundreds of sub-haloes, most of which should have formed small dwarf-like galaxies in their centers. Such objects should in principle be detectable in the outskirts of the large galaxies they will eventually be accreted by.

The long-standing discrepancy between the numbers of presently observed dwarf galaxies surrounding the Milky Way and this expectation is known as the "missing satellite problem" (Diemand et al. 2007, and references therein). The magnitude of this discrepancy has been reduced (but not yet solved) by the discovery of numerous low luminosity dwarf spheroidals (dSphs) by imaging from the Sloan Digital Sky Survey (Belokurov et al. 2006; Zucker et al. 2006; Walsh et al. 2008). Since many of these newly discovered 
dSphs exhibit surface brightnesses at the very limit of what might be detectable from the ground, it remains possible that the missing satellite problem could be completely resolvable, but just beyond our current reach with ground-based observations.

Another expectation from $\Lambda$ CDM-based simulations is that many of the satellites, and their attendant stellar populations, may have already been accreted, and mixed (or partially mixed) into the halo populations of the Milky Way. In order to test this idea, we are required to investigate likely signatures of their presence, as reflected in the kinematics and chemistry of the halo populations. Here we report on a current effort to do just this, based on the large (and growing) body of medium-resolution spectroscopy of stars in the halo observed during the course of the SDSS and its first extension (SDSS-II). Below we present a few of the most pertinent highlights, including the recognition of the dual halo structure of the Galaxy (Carollo et al. 2007), its decomposition into individual components (Carollo et al., in prep.), and the measurement of alpha abundance ratios for many tens of thousands of stars from SDSS (Lee et al., in prep.).
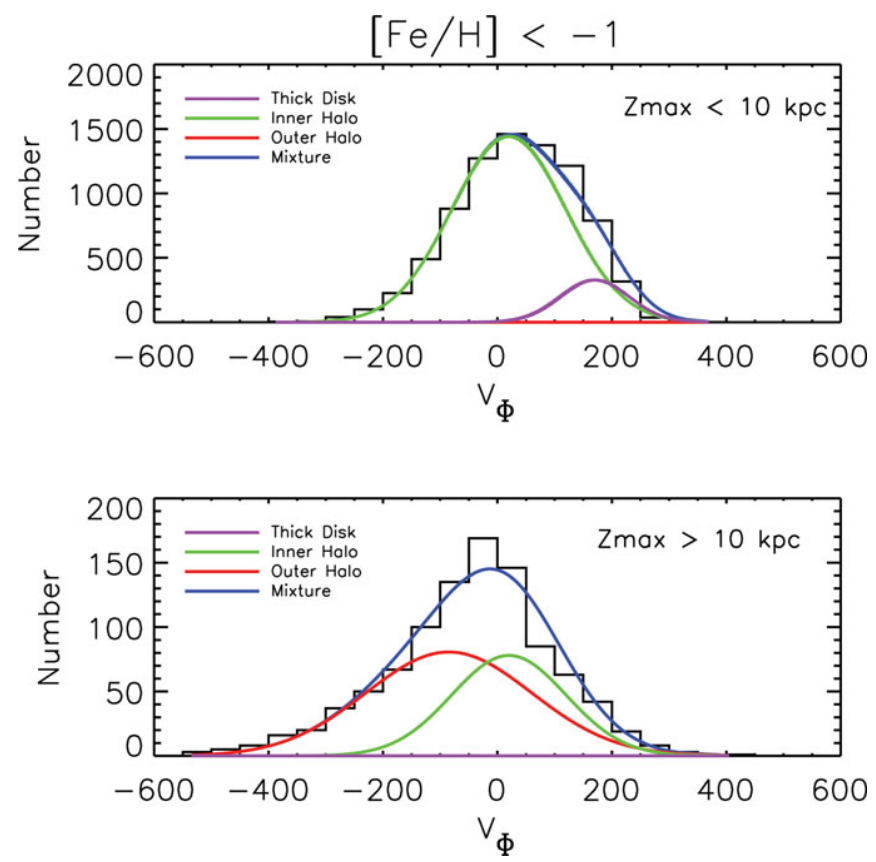

Figure 1. An example maximum-likelihood decomposition of the contribution of thick-disk, inner-halo, and outer-halo populations to the overall observed distribution of $\mathrm{V}_{\Phi}$, the rotation with respect to the Galactic center, based on an analysis of the SDSS/SEGUE calibration stars from DR-6 (Adelman-McCarthy et al. 2008). All stars in the figure have $[\mathrm{Fe} / \mathrm{H}]<-1.0$. The upper panel applies to stars with $\mathrm{Z}_{\max }<10 \mathrm{kpc}$; the lower panel applies to stars with $\mathrm{Z}_{\max }>$ $10 \mathrm{kpc}$, where $Z_{\max }$ refers to the maximum distance from the Galactic plane reached by an individual star during the course of its orbit. Note that the inner-halo population completely dominates the observed distribution in the upper panel, while a higher dispersion, net retrograde outer-halo population is required to account for the extended low velocity tail in the lower panel. 


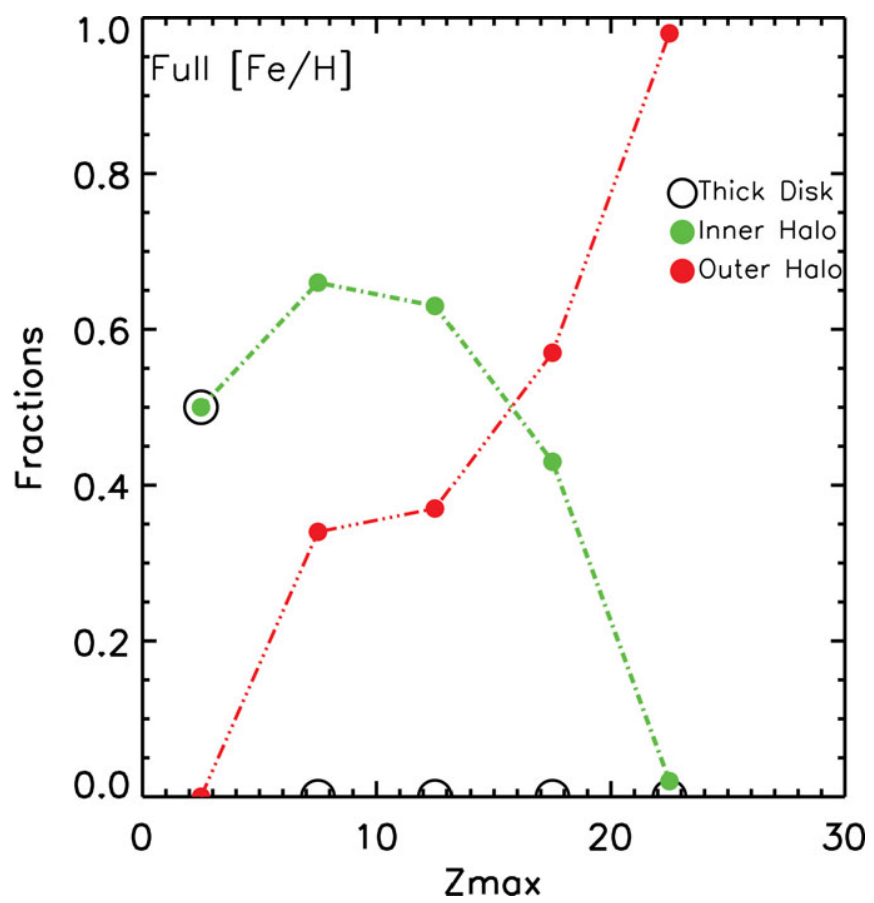

Figure 2. Application of the decomposition models of Carollo et al. (in prep.) in order to estimate the contribution of various populations as a function of $Z_{\mathrm{max}}$, over the entire metallicity range of the SDSS/calibration star sample. The inner halo is indicated by the green line (and dots), while the outer halo is represented by the red line (and dots). The thick disk is represented by open black circles.

\section{The inner/outer halo structure of the Galaxy}

It has been long speculated that the halo of the Milky Way might be structurally complex, and comprise more than a single stellar population. Individual observational signatures that might be explained by a dual halo model have emerged slowly over the past few decades, and have been reported by many authors. This picture has now begun to come into focus. Recently, Carollo et al. (2007) used calibration stars from SDSS/SEGUE to demonstrate convincingly that the halo is indeed clearly divisible into two broadly overlapping structural components, an inner and an outer halo, which exhibit different spatial density profiles, stellar orbits, and stellar metallicities. While the inner halo has a modest net prograde rotation (now estimated to be $20 \pm 5 \mathrm{~km} / \mathrm{s}$ ), the outer halo exhibits a net retrograde rotation (now estimated to be $-85 \pm 9 \mathrm{~km} / \mathrm{s}$ ), and a peak metallicity three times lower $([\mathrm{Fe} / \mathrm{H}]=-2.2)$ than that of the inner halo $([\mathrm{Fe} / \mathrm{H}]$ $=-1.6)$. These properties indicate that the individual halo components likely formed in fundamentally different ways, possibly through successive dissipational (inner) and dissipationless (outer) mergers, and the tidal disruption of proto-Galactic clumps. Work is now in progress to derive the velocity ellipsoids and metallicity distribution functions of the individual populations (see Fig. 1).

The application of these decomposition models to the DR- 6 calibration stars can also be used to derive the approximate fractions of stars contributed to the Galaxy from each stellar population. Fig. 2 indicates that while the thick disk and inner halo dominate 
within $5 \mathrm{kpc}$ of the plane, the inner halo dominates between 5 and $15 \mathrm{kpc}$ from the plane, and the outer halo dominates beyond $15 \mathrm{kpc}$.

\section{The measurement of alpha abundance ratios}

Before estimation of the $[\alpha / \mathrm{Fe}]$ ratios for SDSS/SEGUE stars, one first requires estimates of the fundamental atmospheric parameters $\mathrm{T}_{\mathrm{eff}}, \log \mathrm{g}$, and $[\mathrm{Fe} / \mathrm{H}]$.

In an effort to provide robust determinations that remain valid over the large range of parameter space and $S / N$ explored by SEGUE, the atmospheric parameters are derived from a set of techniques, based on a number of different calibrations. These approaches, which collectively are applied by the SEGUE Stellar Parameter Pipeline (SSPP), include techniques for finding the minimum distance (parameterized in various ways) between observed spectra and grids of synthetic spectra (e.g., Allende Prieto et al. 2006), non-linear regression models (e.g., Re Fiorentin et al. 2007, and references therein), correlations between broadband colors and the strength of prominent metallic lines, such as the CaII K line (Beers et al. 1999), auto-correlation analysis of a stellar spectrum (Beers et al. 1999, and references therein), obtaining fits of spectral lines (or summed line indices) as a function of broadband colors (Wilhelm et al. 1999), or the behavior of the CaII triplet lines as a function of broadband color (Cenarro et al. 2001). Details of these procedures and tests of the validity of the resulting parameter estimates are presented in a series of three papers, Lee et al. (2008a), Lee et al. (2008b), and Allende Prieto et al. (2008), to which the interested reader is referred.
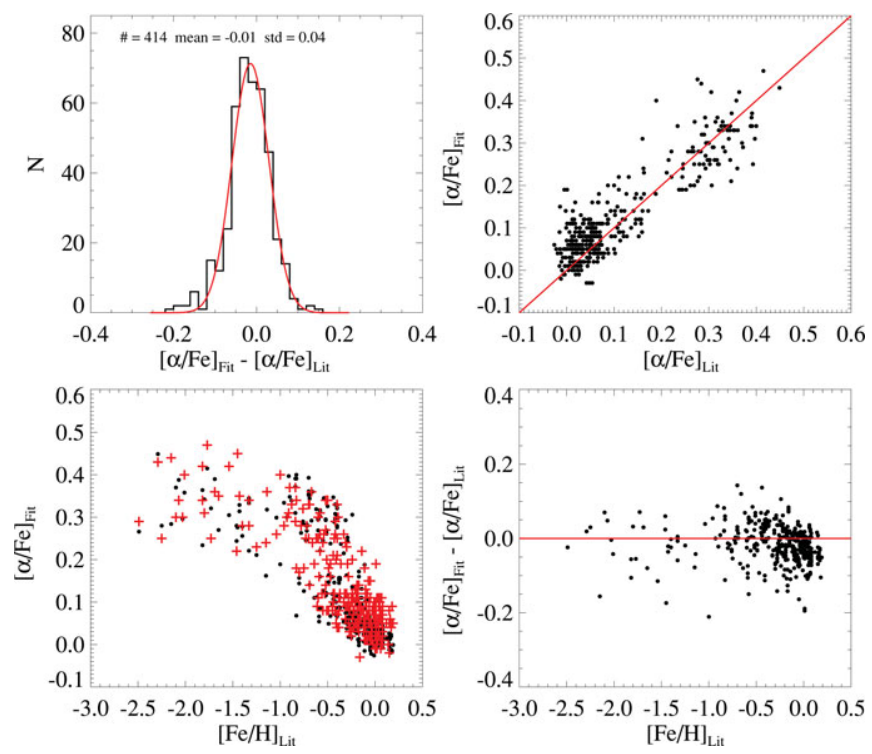

Figure 3. Comparison of $[\alpha / \mathrm{Fe}]$ from our estimates (Fit) with those from the literature (Lit), based on spectra from the ELODIE library (Moultaka et al. 2004). The upper left panel is a histogram of the residuals, overplotted with a Gaussian fit. The red crosses in the bottom left panel are our determinations, while the black dots represent the literature values. Residuals in our determinations as a function of $[\alpha / \mathrm{Fe}]_{\mathrm{Lit}}$ and $[\mathrm{Fe} / \mathrm{H}]_{\mathrm{Lit}}$ are shown in the right-hand panels. 
The precision of the parameter estimates varies with the $S / N$ of the spectra. At the median $S / N$ of the SEGUE spectra (roughly 20/1), the estimates have typical errors of $\delta \mathrm{T}_{\text {eff }}=150 \mathrm{~K}, \delta \log \mathrm{g}=0.30 \mathrm{dex}$, and $\delta[\mathrm{Fe} / \mathrm{H}]=0.25 \mathrm{dex}$, respectively. Tests on the accuracy of the atmospheric parameter estimates indicate that there exist negligible zero-point offsets over most of the parameter space.

Lee et al. (in prep.) describe a spectral fitting method for derivation of $[\alpha / \mathrm{Fe}]$ ratios from medium-resolution spectroscopy, based on a large grid of synthetic spectra covering a range in atmospheric parameters, and with $[\alpha / \mathrm{Fe}]$ ratios in the range $-0.1 \leqslant \alpha / \mathrm{Fe}]$ $\leqslant+0.6$. This approach is validated by comparison with an external library of highresolution spectra (ELODIE; Moultaka et al. 2004), for stars with estimates of alphaelement abundances that have already appeared in the literature.

A total of 414 spectra for stars selected from the ELODIE library were processed in the same way as the synthetic spectra, after degrading them to the SDSS resolving power $R=2000$. Estimates of their $[\alpha / \mathrm{Fe}]$ were obtained by spectral matching to the synthetic spectra grid. Fig. 3 shows the results of the comparison. A Gaussian fit to the residuals between our values and those from the literature indicate that there is little systematic offset, and further indicates a very small scatter (standard deviation of 0.04 dex). No trends in the estimated alpha abundances as a function of $[\mathrm{Fe} / \mathrm{H}]$ are noted for stars with $[\mathrm{Fe} / \mathrm{H}]<-0.5$ in the right-hand panels of this figure.

A noise-injection test indicates that, for SDSS spectra with $S / N>20 / 1,[\alpha / \mathrm{Fe}]$ can be estimated with an rms scatter $<0.1 \mathrm{dex}$ (and negligible zero-point offset), which is sufficiently precise to carry out inspection of the variations of alpha abundances as a function of $[\mathrm{Fe} / \mathrm{H}]$ and other quantities.
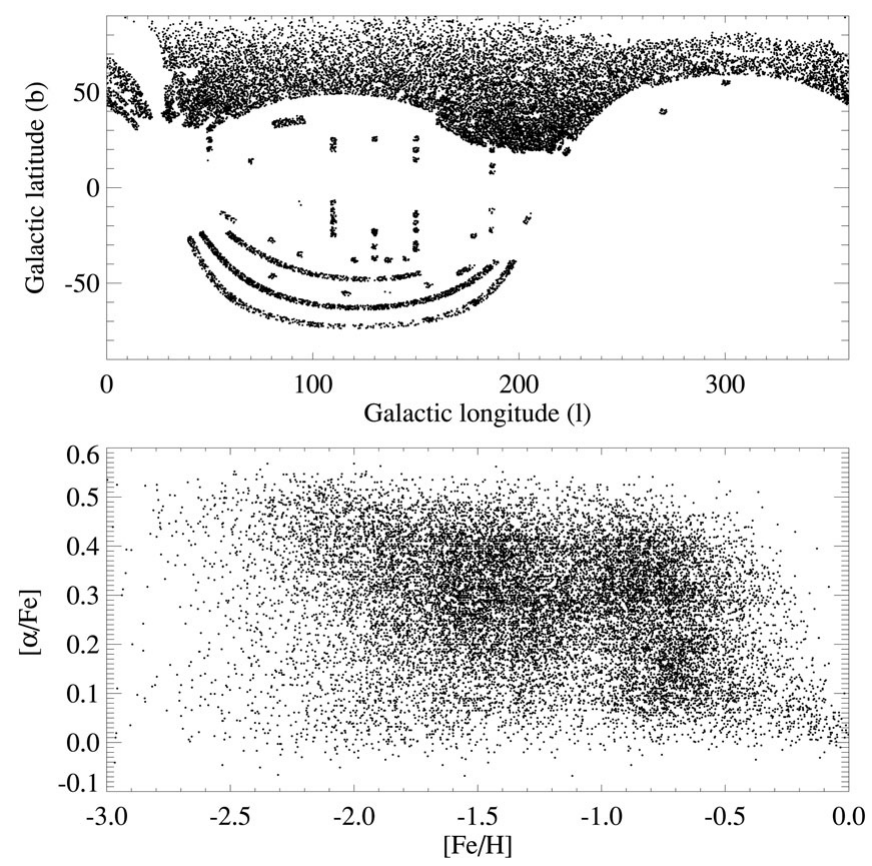

Figure 4. (upper panel) Spatial distribution of the sample (17450 stars) in a Galactic coordinate system. (bottom panel) $[\alpha / \mathrm{Fe}]$ as a function of $[\mathrm{Fe} / \mathrm{H}]$. 

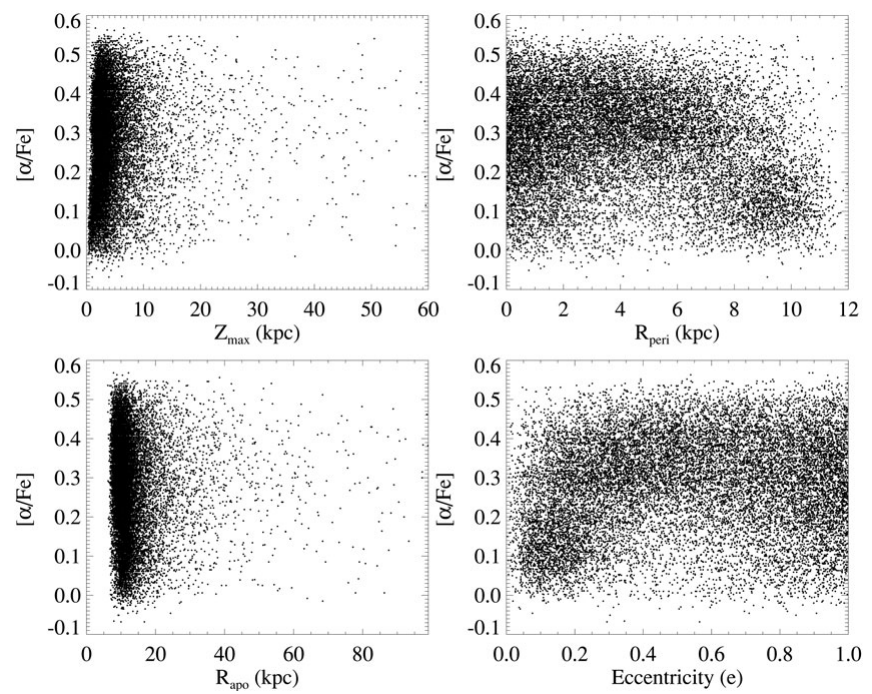

Figure 5. Distribution of $[\alpha / \mathrm{Fe}]$ with respect to $\mathrm{Z}_{\mathrm{max}}, \mathrm{R}_{\mathrm{peri}}, \mathrm{R}_{\mathrm{apo}}$, and orbital eccentricity.

\section{Correlations of alpha abundance ratios with metallicity and orbital parameters}

A total of 39,167 spectrophotometric and telluric calibration stars from SDSS/SEGUE were selected and processed by the SSPP in order to derive their stellar atmosphere parameters. Then their $[\alpha / \mathrm{Fe}]$ ratios were estimated as described above. Signal-to-noise cuts were then applied to this sample, leaving 17,450 stars with alpha-element abundance estimates based on spectra with $S / N>20 / 1$. The spatial distribution of these stars is shown in the upper panel of Fig. 4. The bottom panel of this figure shows the distribution of $[\alpha / \mathrm{Fe}]$ as a function of $[\mathrm{Fe} / \mathrm{H}]$.

At lower metallicities, inspection of Fig. 4 reveals the existence of a plateau with $[\alpha / \mathrm{Fe}]$ $\sim+0.4$, which is a typical value for halo stars with metallicities below -1.0 . Above $[\mathrm{Fe} / \mathrm{H}]=-1.0$, the alpha abundances decrease with metallicity and reach the solar value at $[\mathrm{Fe} / \mathrm{H}]=0$, as expected. It is also clear that there exists a substantial fraction of low-metallicity stars with $[\alpha / \mathrm{Fe}]<+0.2$. At low metallicities, there is a suggestion of a bifurcation of the alpha abundances into two primary groupings, high- and low-alpha stars, with a dearth of stars having intermediate alpha-element enrichment.

Orbital parameters for a smaller subset of the data (those having well-measured proper motions, and passing additional cuts to ensure they belong to a sufficiently local sample that assumptions involved in the derivation of the orbital parameters are satisfied) can be used to examine the distribution of $[\alpha / \mathrm{Fe}]$ with $\mathrm{Z}_{\max }, \mathrm{R}_{\text {peri }}, \mathrm{R}_{\text {apo }}$, and eccentricity. Fig. 5 shows these distributions.

It is difficult to discern any obvious trends in the behavior of the alpha element abundances with orbital parameters; there are certainly regions which appear over- or underpopulated, but it is premature to suggest their causes. However, inspection of these data has only just begun.

It is clear that full understanding of the connection between the measured alpha abundance distributions and models for the formation and evolution of the Galaxy will require comparison with more sophisticated numerical simulations of the galaxy assembly process 
(which take into account chemistry and kinematics), such as those just now becoming available (e.g., Tumlinson et al. 2006; Johnston et al. 2008). It would also be very desirable to obtain higher resolution spectroscopy of targeted inner- and outer-halo stars, from which more accurate estimates of $[\alpha / \mathrm{Fe}]$ ratios could be derived. Several groups have already begun this effort.

\section{Acknowledgements}

Funding for the SDSS and SDSS-II has been provided by the Alfred P. Sloan Foundation, the Participating Institutions, the National Science Foundation, the U.S. Department of Energy, the National Aeronautics and Space Administration, the Japanese Monbukagakusho, the Max Planck Society, and the Higher Education Funding Council for England. The SDSS Web Site is http://www.sdss.org/.

This work received partial support from grants AST 07-07776 and PHY 02-15783; Physics Frontier Center / Joint Institute for Nuclear Astrophysics (JINA), awarded by the US National Science Foundation.

\section{References}

Adelman-McCarthy, J. K, Agüeros, M. A., Allah, S. S, Allende Prieto, C., \& Anderson, K. S. J., et al. 2008, ApJS, 175, 297

Allende Prieto, C., Beers, T. C., Wilhelm, R., Newberg, H. J., \& Rockosi, C. M., et al. 2006, ApJ, 636, 804

Beers, T. C., Rossi, S., Norris, J. E., Ryan, S. G., \& Shefler, T. 1999, AJ, 117, 981

Bekki, K. \& Chiba, M. 2001, ApJ, 558, 666

Allende Prieto, C., Sivarani, T., Beers, T. C., Lee, Y. S., \& Koesterke, L. et al. 2008, AJ, in press (arXiv:0710.5780)

Belokurov, V., Zucker, D. B., Evans, N. W., Gilmore, G., \& Vidrih, S., et al. 2006, ApJ, 642, L137

Carollo, D., Beers, T. C., Lee, Y. S., Chiba, M., \& Norris, J. E., et al. 2007, Nature, 450, 1020

Cenarro, A. J., Cardiel, N, Gorgas, J., Peletier, R. F., Vazdekis, A., \& Prada, F. 2001, MNRAS, 326,959

Diemand, J., Kuhlen, M., \& Madau, P. 2007 2007, ApJ, 667, 859

Johnston, K. V., Bullock, J. S., Sharma, S., Font, A., Robertson, B. E., \& Leitner, S. N. 2008, ApJ, in press (arXiv:0807.3911)

Lee, Y. S., Beers, T.C., Sivarani, T., Allende Prieto, C., \& Koesterke, L., et al. 2008, AJ, in press (arXiv:0710.5645)

Lee, Y. S., Beers, T. C., Sivarani, T., Johnson, J. A., \& An, D., et al. 2008, AJ, in press (arXiv:0710.5778)

Moultaka, J., Ilovaisky, S. A., Prugniel, P., \& Soubiran, C. 2004, PASP, 116, 693

Re Fiorentin, P., Bailer-Jones, C. A. L., Lee, Y. S., Beers, T. C., \& Sivarani, T., et al. 2007, A\&SA, 467, 1373

Tumlinson, J. 2006, ApJ, 641, 1

Walsh, S. M., Willman, B., \& Jerjen, H. 2008, AJ, submitted (arXiv:0807.3345)

Wilhelm, R., Beers, T. C., \& Gray, R. O. 1999, AJ, 117, 2308

Zucker, D. B., Belokurov, V., Evans, N. W., Wilkinson, M. I., \& Irwin, M. J., et al. 2006, ApJ, 643, L103 